\title{
Affibody scaffolds improve sesquiterpene production in
}

\section{Saccharomyces cerevisiae}

Stefan Tippmann ${ }^{1,2^{*}}$, Josefine Anfelt ${ }^{3^{*}}$, Florian David ${ }^{1,2}$, Jacqueline M. Rand ${ }^{1,4}$, Verena Siewers ${ }^{1,2}$, Mathias Uhlén $^{3,5}$, Jens Nielsen ${ }^{1,2,5}$, and Elton P. Hudson ${ }^{3 * *}$

${ }^{1}$ Department of Biology and Biological Engineering, Chalmers University of Technology, SE412 96 Gothenburg, Sweden

${ }^{2}$ Novo Nordisk Foundation Center for Biosustainability, Chalmers University of Technology, SE412 96 Gothenburg, Sweden

${ }^{3}$ Division of Proteomics and Nanobiotechnology, School of Biotechnology, Royal Institute of Technology (KTH), Science for Life Laboratory, SE171 21 Stockholm, Sweden

${ }^{4}$ Department of Chemical and Biological Engineering, University of Wisconsin-Madison, Madison, WI 53706, USA

${ }^{5}$ Novo Nordisk Foundation Center for Biosustainability, Technical University of Denmark, DK2970 Hørsholm, Denmark

*Equal contribution

**Corresponding Author: Elton P. Hudson

Division of Proteomics and Nanobiotechnology, School of Biotechnology, Royal Institute of Technology (KTH), Science for Life Laboratory, SE171 21 Stockholm, Sweden

E-mail: paul.hudson@scilifelab.se

Tel: +46855378317

Keywords: affibodies, isoprenoids, biofuels, PHB, yeast, metabolic engineering 
Supplementary Table 1: List of primers used for plasmid construction. Bold letters indicate restriction sites, italics indicate overhangs.

\begin{tabular}{|c|c|c|c|}
\hline Plasmid & Primer & Sequence $\left(5^{\prime} \rightarrow 3^{\prime}\right)$ & Template \\
\hline \multirow{2}{*}{ pAffiRef } & Erg20-5D-fwd & GTTGTTGGATCCAAAACAATGGCTTCAGAAAAAGAAATTA & \multirow{2}{*}{$\begin{array}{l}\text { CEN.PK } \\
113-5 D \\
\end{array}$} \\
\hline & Erg20-5D-rev & GTTGTTCTCGAGCTATTTGCTTCTCTTGTAAACTTT & \\
\hline \multirow{12}{*}{ pAffi1 } & pSPGM1-fwd & AGGAGTTAGACAACCTGAAGTC & \multirow{2}{*}{ pAffiRef } \\
\hline & pSPGM1-rev & GCCTTATTATTGACAGAACC & \\
\hline & FarnSyn-fwd & TATTAGATAGCCAATGGTTCTGT & \multirow{2}{*}{ pAffiRef } \\
\hline & FarnSyn-rev & TCTTCCGGTTCCAGTTCCAGCGGCAAAGATATGTCCATCCCATTATTA & \\
\hline & ZTaq-fwd & GCCGCTGGAACTGGAACC & \multirow{2}{*}{ pAff8c_PHB_Z } \\
\hline & ZTaq-rev & ATCTAATCTAAGTTTTAATTACAAAAAACAATGGTAGACAACAAATTCAACAAA & \\
\hline & Pro-fwd & TTGTAATTAAAACTTAGATTAGATT & \multirow{2}{*}{ pAffiRef } \\
\hline & Pro-rev & TGTTTCTTTGTTGAATTTGTTGTCTACCATTGTTTTTTGTTTTATATTTGTTGTAA & \\
\hline & ZlgA-fwd & ATGGTAGACAACAAATTCAACA & \multirow{2}{*}{ pAff8c_PHB_Z } \\
\hline & ZlgA-rev & TСTCTCTCTCCTAATTTCTTTTTCTGAAGCACCAGAACTACTGGAGCCGC & \\
\hline & Erg20-fwd & GCTTCAGAAAAAGAAATTAGGAGAG & \multirow{2}{*}{ pAffiRef } \\
\hline & Erg20-rev & CTAGACTTCAGGTTGTCTAACTCCT & \\
\hline \multirow{6}{*}{ pAffi5 } & Affi5-1-fwd & ACAGCCAAGCTTTTATTTTCGGC & \multirow{2}{*}{ pAffi2 } \\
\hline & Affi5-1-rev & CTAAAGGGAACAAAAGCTGGAGCTCGCACACACCATAGCTTCAAAATG & \\
\hline & Affi5-2-fwd & CGCCGAAATAAAAGCTTGGCTGTTCGAATTTACGTAGCCCAAT & CEN.PK \\
\hline & Affi5-2-rev & GGCGAATTGGGTACCGGCCGATGGTAATGATCCGAACTTG & 113-5D \\
\hline & p416TEF4-fwd & GAGCTCCAGCTTTTTGTTCCCTT & \multirow{2}{*}{ p416TEF } \\
\hline & p416TEF4-rev & GGCCGGTACCCAATTCG & \\
\hline \multirow{6}{*}{ pAffi3 } & Affi3-1-rev & GCGTTTATCCAGCTGCATTAATGAGCACACACCATAGCTTCAAAATG & \multirow{2}{*}{ pAffi5 } \\
\hline & Affi3-1-fwd & CCTGATTCTGTGGATAACCGTATTACCGATGGTAATGATCCGAACTTG & \\
\hline & Affi3-2-fwd & GGTAATACGGTTATCCACAGAATC & \multirow{2}{*}{ pAffi1 } \\
\hline & Affi3-2-rev & TTCTCAGGTATAGCATGAGGTC & \\
\hline & Affi3-3-fwd & GAGCGACCTCATGCTATACCT & \multirow{2}{*}{ pAffi1 } \\
\hline & Affi3-3-rev & TCATTAATGCAGCTGGATAAAC & \\
\hline
\end{tabular}




\begin{tabular}{|c|c|c|c|}
\hline \multirow{6}{*}{ pAffi7 } & Affi5-1-rev2 & ATGGTAGACAACAAATTCAACAAAGAAAGAG & \multirow{2}{*}{ pAffi5 } \\
\hline & Affi5-2-rev & GGCGAATTGGGTACCGGCCGATGGTAATGATCCGAACTTG & \\
\hline & PAdh1-fwd & CTAAAGGGAACAAAAGCTGGAGCTCAGGGGGATCGAAGAAATGAT & \multirow{2}{*}{$\begin{array}{l}\text { CEN.PK } \\
113-5 D\end{array}$} \\
\hline & PAdh1-rev & TTTGTTGAATTTGTTGTCTACCATTGTTTTTGTATATGAGATAGTTGATTGTATGCTT & \\
\hline & p416TEF4-fwd & GAGCTCCAGCTTTTTGTTCCCTT & \multirow{2}{*}{ p416TEF } \\
\hline & p416TEF4-rev & GGCCGGTACCCAATTCG & \\
\hline \multirow{6}{*}{ pAffi8 } & Affi5-1-rev2 & ATGGTAGACAACAAATTCAACAAAGAAAGAG & \multirow{2}{*}{ pAffi5 } \\
\hline & Affi5-2-rev & GGCGAATTGGGTACCGGCCGATGGTAATGATCCGAACTTG & \\
\hline & PKex2-fwd & CTAAAGGGAACAAAAGCTGGAGCTCGTAGATACACGTATCTCGACATG & \multirow{2}{*}{$\begin{array}{l}\text { CEN.PK } \\
113-5 D\end{array}$} \\
\hline & PKex2-rev & TTTGTTGAATTTGTTGTCTACCATTGTTTTATCTGATAATGGGTTAGTAGTTTATAAT & \\
\hline & p416TEF4-fwd & GAGCTCCAGCTTTTTGTTCCCTT & \multirow{2}{*}{ p416TEF } \\
\hline & p416TEF4-rev & GGCCGGTACCCAATTCG & \\
\hline \multirow{6}{*}{ pAffi9 } & Affi9-1-rev & GCGTTTATCCAGCTGCATTAATGAAGGGGGATCGAAGAAATGAT & \multirow{2}{*}{ pAffi7 } \\
\hline & Affi3-1-fwd & CCTGATTCTGTGGATAACCGTATTACCGATGGTAATGATCCGAACTTG & \\
\hline & Affi3-2-fwd & GGTAATACGGTTATCCACAGAATC & \multirow{2}{*}{ pAffi1 } \\
\hline & Affi3-2-rev & TTCTCAGGTATAGCATGAGGTC & \\
\hline & Affi3-3-fwd & GAGCGACCTCATGCTATACCT & \multirow{2}{*}{ pAffi1 } \\
\hline & Affi3-3-rev & TCATTAATGCAGCTGGATAAAC & \\
\hline \multirow{6}{*}{ pAffi10 } & Affi10-1-rev & GCGTTTATCCAGCTGCATTAATGAGTAGATACACGTATCTCGACATG & \multirow{2}{*}{ pAffi8 } \\
\hline & Affi3-1-fwd & CCTGATTCTGTGGATAACCGTATTACCGATGGTAATGATCCGAACTTG & \\
\hline & Affi3-2-fwd & GGTAATACGGTTATCCACAGAATC & \multirow{2}{*}{ pAffi1 } \\
\hline & Affi3-2-rev & TTCTCAGGTATAGCATGAGGTC & \\
\hline & Affi3-3-fwd & GAGCGACCTCATGCTATACCT & \multirow{2}{*}{ pAffi1 } \\
\hline & Affi3-3-rev & TCATTAATGCAGCTGGATAAAC & \\
\hline
\end{tabular}




\begin{tabular}{|c|c|c|c|}
\hline \multirow{6}{*}{ pAffi11 } & Affi11-1-fwd & GCCGCTAGACGAGCTGCC & \multirow{2}{*}{ pAffi5 } \\
\hline & Affi11-1-rev & TGCGTTATCCCCTGATTCTGT & \\
\hline & Affi11-2-fwd & GAGATAGGGTTGAGTGTTGTTC & \multirow{2}{*}{ pAffis } \\
\hline & Affi11-2-rev & CAGCTCCGGCAGCTCGTCTAGCGGCGTAGACAACAAATTCAACAAAGAAGC & \\
\hline & Affi11-3-fwd & CTCAAAGGCGGTAATACGGT & \multirow{2}{*}{ pAffi5 } \\
\hline & Affi11-3-rev & TTCTTTAATAGTGGACTCTTGTTCC & \\
\hline \multirow{6}{*}{ pAffi12 } & Affi12-1-fwd & GCCGGAGCTGGACGATTT & \multirow{2}{*}{ pAffi5 } \\
\hline & Affi11-1-rev & TGCGTTATCCCCTGATTCTGT & \\
\hline & Affi11-2-fwd & GAGATAGGGTTGAGTGTTGTTC & \multirow{2}{*}{ pAffi5 } \\
\hline & Affi12-2-rev & CGAAATCGTCCAGCTCCGGCGTAGACAACAAATTCAACAAAGAAGC & \\
\hline & Affi11-3-fwd & CTCAAAGGCGGTAATACGGT & \multirow{2}{*}{ pAffi5 } \\
\hline & Affi11-3-rev & TTCTTTAATAGTGGACTCTTGTTCC & \\
\hline
\end{tabular}

pAffi2 expresses fusion of anti- $\mathrm{Z}_{\text {Taq }}$ and anti- $\mathrm{Z}_{\mathrm{IgA}}$ from $\mathrm{P}_{\text {TEF1 }}$ in plasmid pIYC04. ${ }^{1}$ 
Supplementary Table 2: Primers used for integration of farnesene synthase and FPP synthase as well as their fusions to affibodies $Z_{\text {Taq }}$ and $Z_{\text {IgA }}$ respectively for construction of strains ScAffi01, 03, 07 and 09.

\begin{tabular}{llc} 
Primer & Sequence $\left(5^{\prime} \rightarrow 3^{\prime}\right)$ & Template \\
\hline FS_tHMG1-fwd & GAGCGACCTCATGCTATACCTG & pAffiRef \\
FS_tHMG1-rev & CTTCGAGCGTCCCAAAAC & \\
FS_tHMG1-fwd & GAGCGACCTCATGCTATACCTG & pAffi1 \\
FS_tHMG1-rev & CTTCGAGCGTCCCAAAAC & \\
p0390_up-fwd & AGTTACTTGCTCTATGCGTTTGC & \\
p0390_up-rev & GTAGATACGTTGTTGACACTTCTAA & pCfb0390 \\
p0390_down-fwd & ATCCGCTCTAACCGAAAAGG & \\
p0390_down-rev & TGAGAATCCGGACCAGCA & \\
\hline
\end{tabular}

DNA sequences $\left(5^{\prime} \rightarrow 3^{\prime}\right)$ of anti-idiotypic affibody pairs used in this study. Start and stop codons are underlined, $(\text { SSSSG) })_{4}$ linker is highlighted in grey.

$\mathbf{Z}_{\text {Taq: }}$

ATGGTAGACAACAAATTCAACAAAGAACTGGGTTGGGCGACCTGGGAGATCTTCAACTTACCTAACTTAAACGGTGTGCAAGTGAAGGCCTTCATCGATAGTTTA CGGGATGACCCAAGCCAAAGCGCTAACTTGCTAGCAGAAGCTAAAAAGCTAAATGATGCTCAGGCGCCGAAATAA

\section{$Z_{\text {IgA: }}$}

ATGGTAGACAACAAATTCAACAAAGAAACAATACAAGCGAGTCAAGAGATCAGACTATTACCTAACTTAAACGGTAGACAAAAGCTTGCCTTCATCCACAGTTTAC TTGATGACCCAAGCCAAAGCGCTAACTTGCTAGCAGAAGCTAAAAAGCTAAATGATGCTCAGGCGCCGAAATAA

Anti-Z $\mathrm{Z}_{\text {Taq }}$-anti-Z $\mathrm{Z}_{\mathrm{IgA}}$ Scaffold with (SSSSG) ${ }_{4}$ linker:

ATGGTAGACAACAAATTCAACAAAGAAAGAGTGATTGCGATAGGTGAGATCATGCGGTTACCTAACTTAAACAGTCTCCAAGTGGTGGCCTTCATCAATAGTTTA CGGGATGACCCAAGCCAAAGCGCTAACTTGCTAGCAGAAGCTAAAAAGCTAAATGATGCTCAGGCGCCGAAATCGTCCAGCTCCGGCAGCTCGTCTAGCGGCAG CAGCTCTTCCGGTTCCAGTTCCAGCGGCGTAGACAACAAATTCAACAAAGAAGCACAAACGGCGGGGGTGGAGATCATGGAGTTACCTAACTTAAACACCCGGC AACTGCTGGCCTTCATCCAGAGTTTACGAGATGACCCAAGCCAAAGCGCTAACTTGCTAGCAGAAGCTAAAAAGCTAAATGATGCTCAGGCGCCGAAATAA 


\section{$\mathrm{Z}_{\mathrm{WT}}$ :}

ATGGTAGACAACAAATTCAACAAAGAACAACAAAACGCGTTCTATGAGATCTTACATTTACCTAACTTAAACGAAGAACAACGAAACGCCTTCATCCAAAGTTTAA AAGATGACCCAAGCCAAAGCGCTAACTTGCTAGCAGAAGCTAAAAAGCTAAATGATGCTCAGGCGCCGAAATAA

\section{$\mathbf{Z}_{\text {HER2: }}$}

ATGGTAGACAACAAATTCAACAAAGAAATGCGAAACGCGTATTGGGAGATCGCTCTGTTACCTAACTTAAACAATCAGCAAAAGCGCGCCTTCATCCGCAGTTTAT ATGATGACCCAAGCCAAAGCGCTAACTTGCTAGCAGAAGCTAAAAAGCTAAATGATGCTCAGGCGCCGAAATAA

\section{Anti- $\mathrm{Z}_{\mathrm{WT}}$-anti-Z $\mathrm{Z}_{\mathrm{HER} 2}$-anti- $\mathrm{Z}_{\mathrm{IgA}}$ scaffold with (SSSSG) ${ }_{4}$ linkers:}

ATGCATCATCACCACCATCACTCAACTGTAGATAACAAATTTAACAAAGAGACTCAGGAAGCCTCTTGGGAGATCTTCACACTGCCCAACCTGAACGGGCGCCAGG TCGCTGCATTTATTAGCAGCCTGCTGGATGATCCGTCTCAGAGTGCAAACCTGTTAGCAGAGGCCAAGAAGCTGAACGATGCACAAGCACCGAAAAGCTCCAGTT CGGGTAGCAGTTCCAGTGGTTCAAGCAGCTCAGGTTCGAGCTCTTCCGGCGTCGATAATAAGTTCAACAAAGAACGCCGTATGGCTGCGTACGAAATTATTGATC

TTCCGAACTTAAACTGGTTTCAGCTCGAGGCCTTTATTACCAGCCTGAGTGATGATCCTTCTCAGTCTGCGAACCTGTTAGCGGAAGCCAAGAAATTGAACGATGC GCAAGCGCCCAAATCTTCAAGCTCTGGTAGCTCGTCGTCTGGGTCTTCAAGTTCCGGCAGTTCGAGCTCCGGAGTTGACAACAAATTTAACAAGGAAGCGCAAAC TGCGGGTGTGGAAATCATGGAACTGCCAAACCTGAATACACGGCAGCTCCTGGCTTTCATCCAGTCATTACGTGATGATCCGTCCCAGTCAGCGAATTTGCTGGCG GAGGCTAAAAAGCTCAACGATGCGCAAGCTCCTAAAACTAGTIAA

Italics: 6 His tag, Orange: Anti-Z $Z_{W T}$, Blue: anti-Z ${ }_{\text {HeR2 }}$, Green: Anti-Z IgA. Gray: Linkers (SSSSG) 

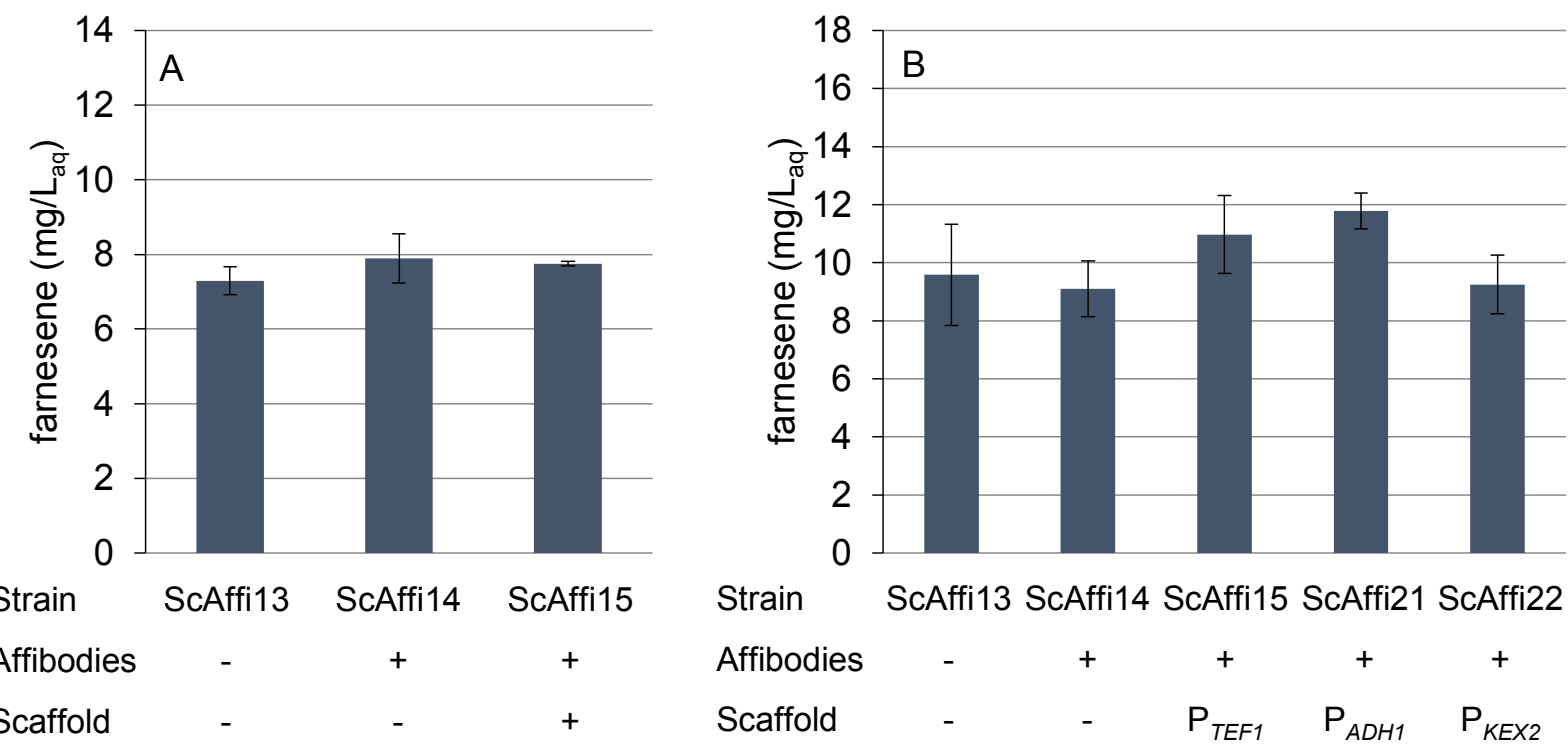

Supplementary Figure 1: Affibody scaffold expression for fusion of FPP and farnesene synthase.

Expression of farnesene and FPP synthase, fused to affibodies $Z_{\text {Taq }}$ and $Z_{\mathrm{IgA}}$, and the anti- $Z_{\mathrm{Taq}}$-anti- $Z_{\mathrm{IgA}}$ scaffold from a single multi-copy plasmid. Scaffold was expressed under promoters (A) $P_{T E F 1}$ and (B) $P_{A D H 1}$ and $\mathrm{P}_{K E X 2}$. Bars represent average farnesene concentrations per volume of aqueous medium of three biological replicates with standard deviations after $72 \mathrm{~h}$ of cultivation in shake flasks.

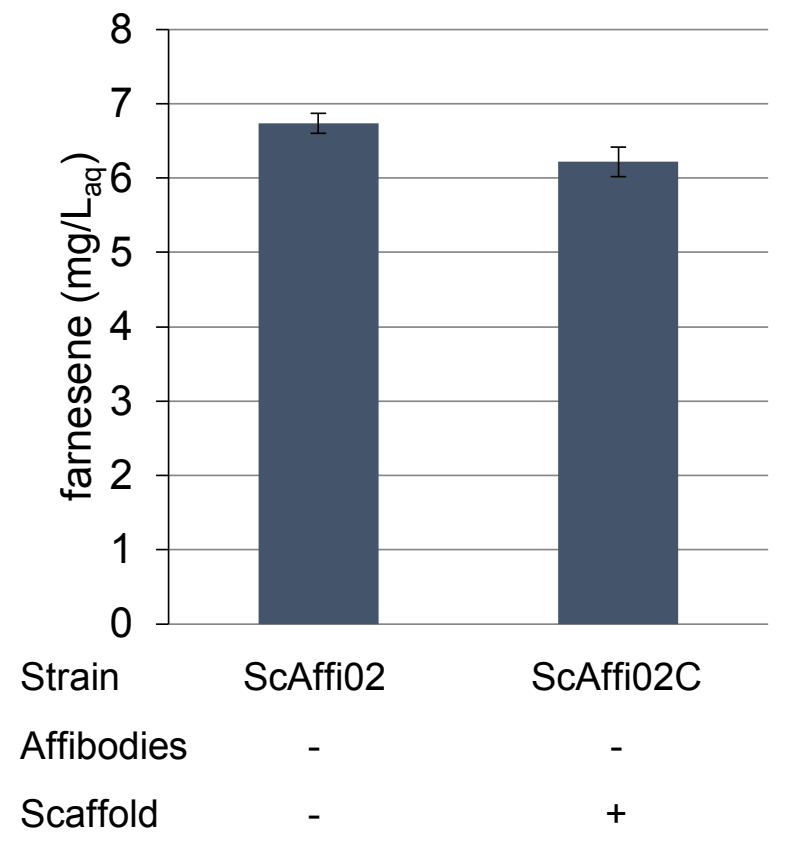

Supplementary Figure 2: Negative control experiment. Effect of scaffold expression (anti- $Z_{\text {IgA }}$, anti- $Z_{\text {Taq }}$ ) on the production of farnesene without expression of their targets $\left(Z_{\operatorname{lgA}}, Z_{\text {Taq }}\right)$. ScAffiO2C expresses scaffold 
from plasmid pAffi5. Bars represent average farnesene concentrations per volume of aqueous medium of three biological replicates with standard deviations after $72 \mathrm{~h}$ of cultivation time in shake flasks.
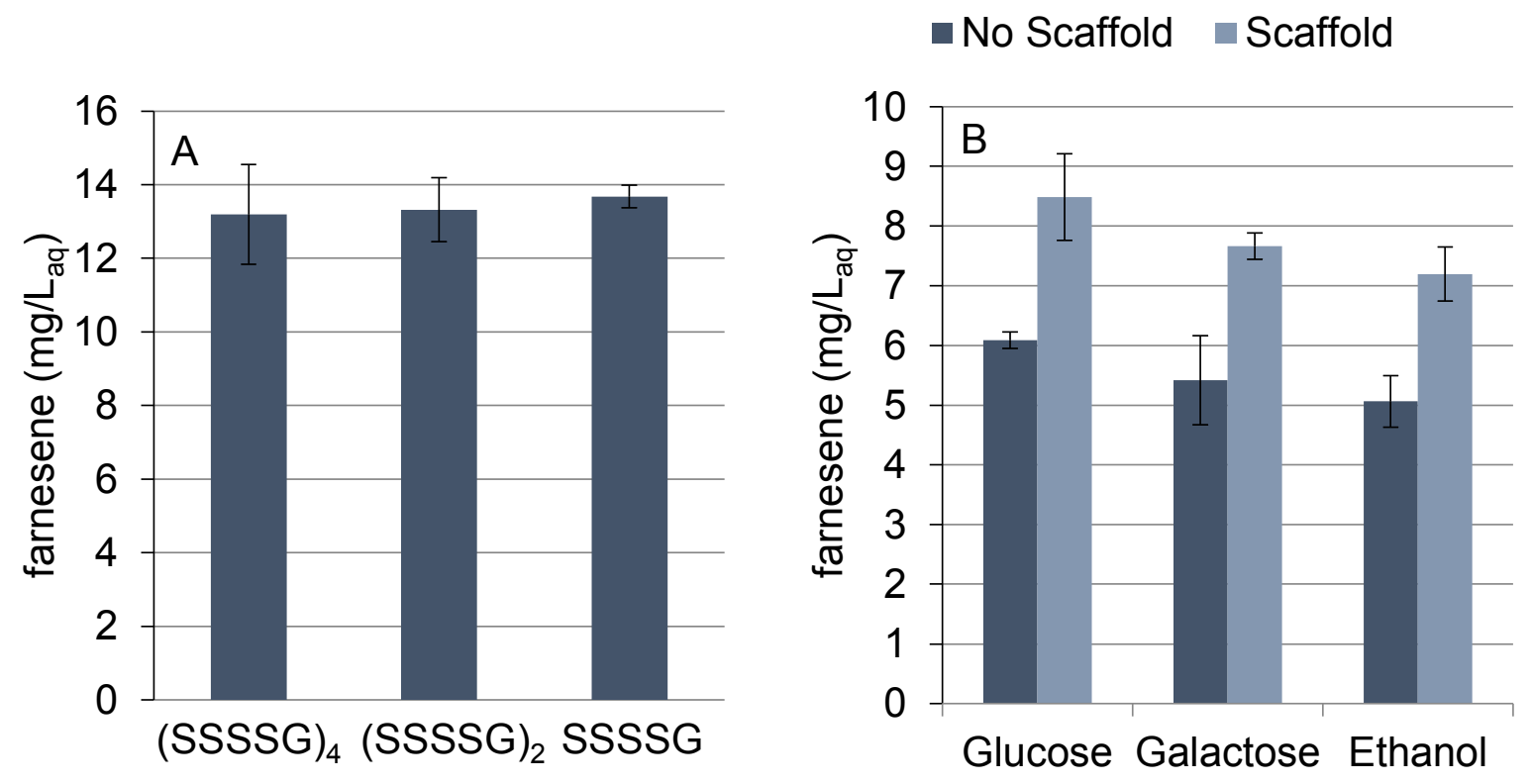

Supplementary Figure 3: Effect of different linker lengths and carbon sources on affibody mediated enzyme fusion for production of farnesene. (A) Linker length between the scaffold affibodies was reduced from 20 to 10 and 5 amino acids. All strains express affibody-enzyme fusions from the chromosome and the scaffold consisting of anti- $Z_{\text {Taq }}$ and anti- $Z_{\operatorname{lgA}}$ from a low copy number plasmid. $(\text { SSSSG) })_{4}$ - ScAffi05, (SSSSG) 2 - ScAffi23 and (SSSSG) 2 - ScAffi24. (B) Effect of scaffold expression with respect to different carbon sources. Cultivations were performed using $1 \% \mathrm{w} / \mathrm{v}$ glucose, galactose and ethanol. No scaffold - ScAffi04, Scaffold - ScAffi05. Bars represent average farnesene concentrations per volume of aqueous medium of three biological replicates with standard deviations after $72 \mathrm{~h}$ of cultivation in shake flasks. 

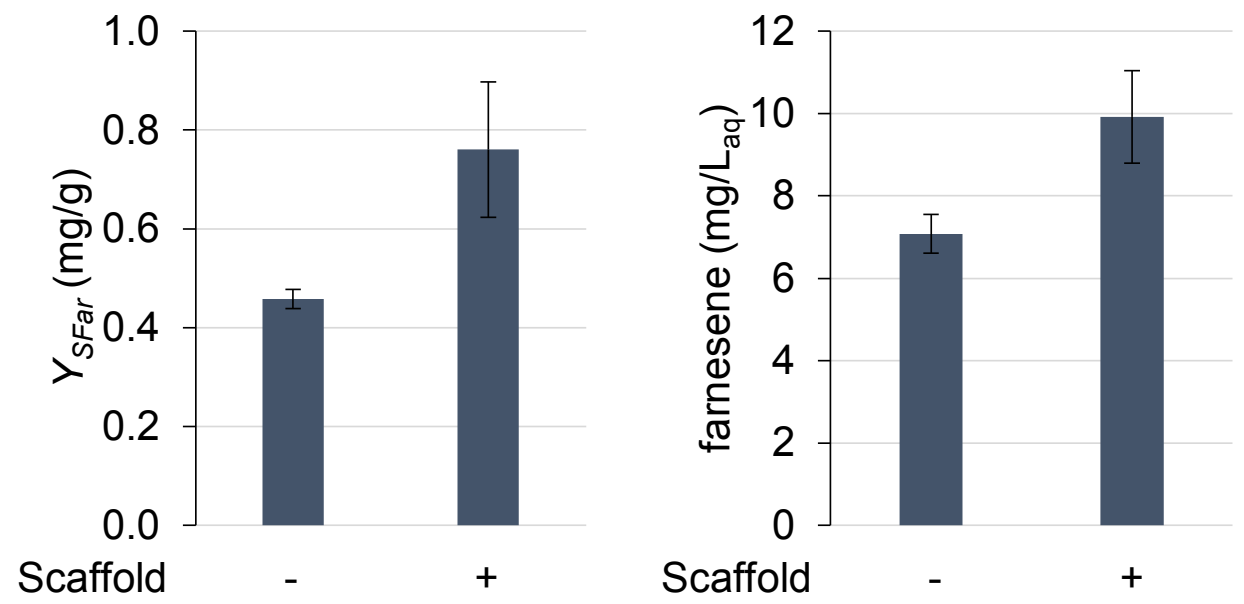

Supplementary Figure 4: Expression of the affibody-scaffold complex for production of farnesene in fedbatch cultivations. All strains express the fusion constructs of affibodies $Z_{\text {Taq }}$ and $Z_{\mathrm{IgA}}$ to the FPP and farnesene synthase from the chromosome. The anti- $Z_{T a q}$-anti- $Z_{\text {IgA }}$ scaffold was expressed from a low copy number plasmid. No scaffold - ScAffi10, scaffold - ScAffi11 (scaffold expression under $\mathrm{P}_{\text {TEF1 }}$ ). Data represent average values of two or three biological replicates with standard deviation $6 \mathrm{~h}$ after initiation of the feed phase.

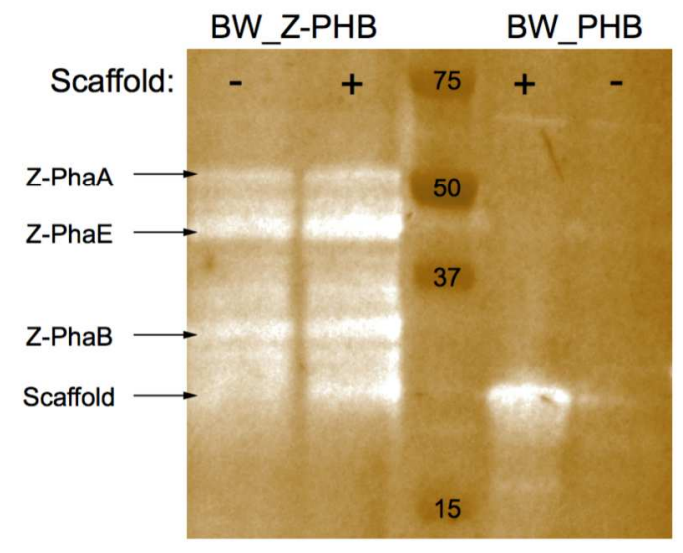

Supplementary Figure 5: Western blot detection of Z-fused enzymes and affibody scaffold in E. coli cell lysates. BW_Z-PHB - strain expressing Z-tagged PHB pathway enzymes, BW_PHB - expression of untagged enzymes. 

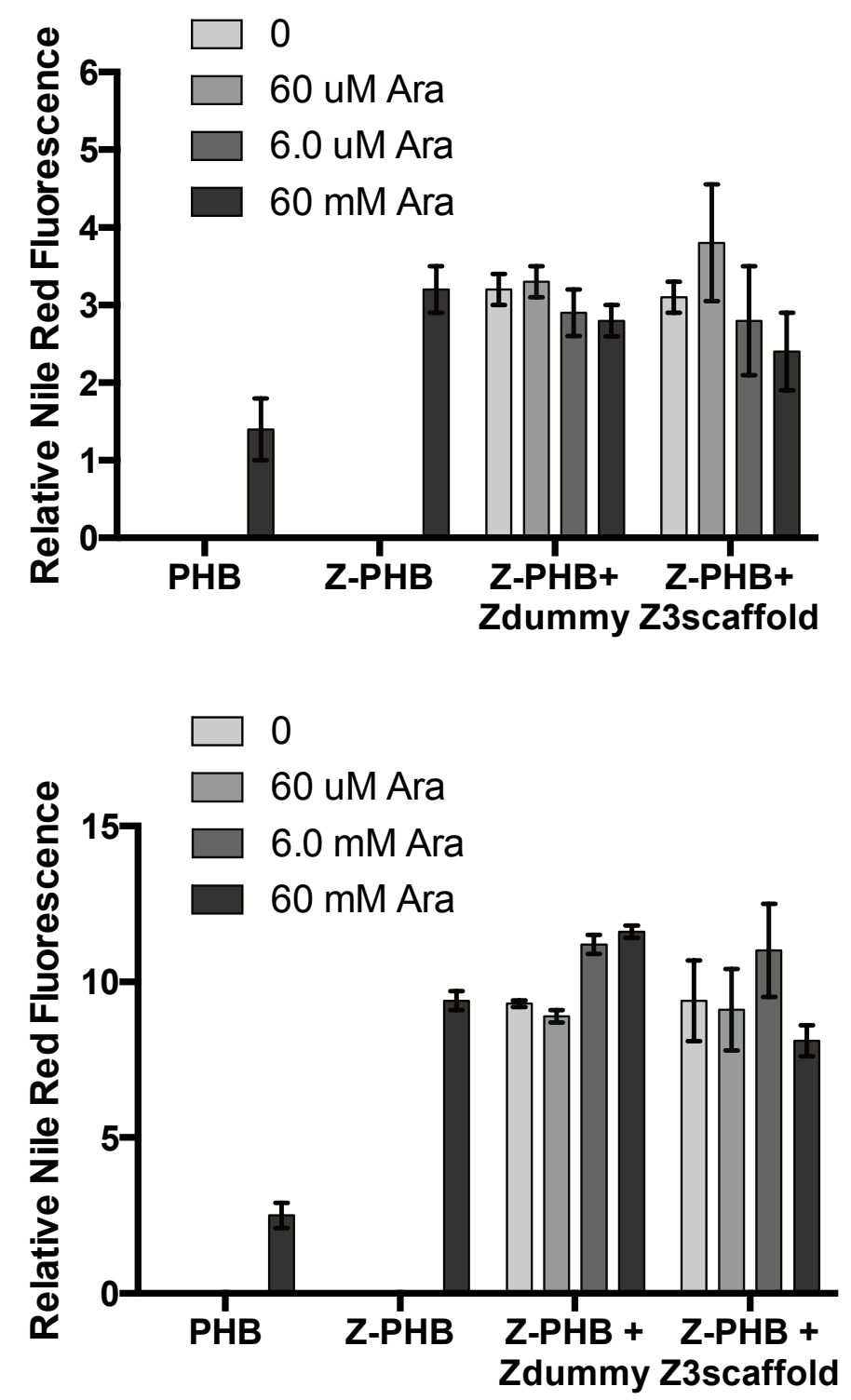

Supplementary Figure 6: Affibody tagging and affibody scaffold effect on PHB production from $E$. coli in LB media $24 \mathrm{hr}$ after induction. Top: Z-PHB induced at $0.05 \mathrm{mM}$ IPTG (low induction). Bottom: Z-PHB induced at $0.5 \mathrm{mM}$ IPTG (high induction). 
A

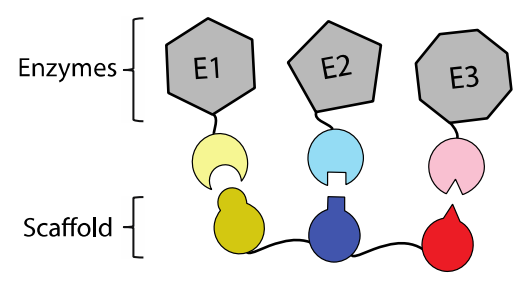

B

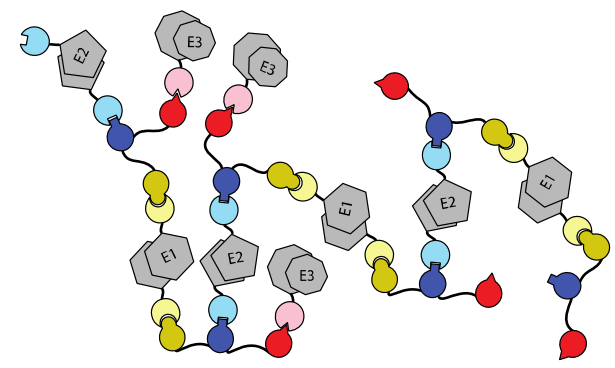

Supplementary Figure 7: (A) Representation of three monomeric enzymes colocalized by a scaffold. (B) An example of how homodimeric enzymes, where there are two tags per enzymes, can coordinate multiple scaffolds, leading to scaffold aggregates.

\section{Supplemental References}

(1) Chen, Y., Daviet, L., Schalk, M., Siewers, V., and Nielsen, J. (2013) Establishing a platform cell factory through engineering of yeast acetyl-CoA metabolism, Metab Eng 15, 48-54.

(2) Jensen, N. B., Strucko, T., Kildegaard, K. R., David, F., Maury, J., Mortensen, U. H., Forster, J., Nielsen, J., and Borodina, I. (2013) EasyClone: Method for iterative chromosomal integration of multiple genes in Saccharomyces cerevisiae, FEMS Yeast Research. 